\title{
Membrane condenser as emerging technology for water recovery and gas pre- treatment: current status and perspectives
}

\author{
Adele Brunetti (D), Francesca Macedonio*, Giuseppe Barbieri and Enrico Drioli
}

\begin{abstract}
The recent roadmap of SPIRE initiative includes the development of "new separation, extraction and pre-treatment technologies" as one of the "key actions" for boosting sustainability, enhancing the availability and quality of existing resources. Membrane condenser is an innovative technology that was recently investigated for the recovery of water vapor for waste gaseous streams, such as flue gas, biogas, cooling tower plumes, etc. Recently, it has been also proposed as pre-treatment unit for the reduction and control of contaminants in waste gaseous streams ( $\mathrm{SO}_{x}$ and $\mathrm{NO}_{x}, \mathrm{VOCs}, \mathrm{H}_{2} \mathrm{~S}, \mathrm{NH}_{3}$, siloxanes, halides, particulates, organic pollutants).

This perspective article reports recent progresses in the applications of the membrane condenser in the treatment of various gaseous streams for water recovery and contaminant control. After an overview of the operating principle, the membranes used, and the main results achieved, the work also proposes the role of this technology as pre-treatment stage to other separation technologies. The potentialities of the technology are also discussed aspiring to pave the way towards the development of an innovative technology where membrane condenser can cover a key role in redesigning the whole upgrading process.
\end{abstract}

Keywords: Pre-treatment, Resources recovery, Waste gases, Membrane engineering

\section{Introduction}

Water is used in the industry for many purposes including processing, washing, diluting, heating, cooling, etc. Across Europe, water abstraction from surface water resources accounts for $58 \%$ of total water use, with the remaining $42 \%$ coming from ground water. Industrial water withdrawals account for ca. $22 \%$ of global water consumption [1]. Its largest single use is in power generation, cooling towers and many other processes, which need water for boilers, cooling and cleaning purposes. In other processes, water is also "produced" (by reactions, in power generation from fossil fuels, biomass, biogas upgrading, etc.) or it is "released" as natural source (geothermal plants). Even though recently industry has shown a significant decrease $(28 \%)$ in water abstraction [2], the long-term vision of the 7th Environment Action Program $[3,4]$ is of an innovative economy in which natural resources are sustainably managed. This includes

\footnotetext{
* Correspondence: a.brunetti@itm.cnr.it; f.macedonio@itm.cnr.it National Research Council of Italy - Institute on Membrane Technology (CNR-ITM), Via Pietro Bucci, cubo 17C, 87036 Rende, CS, Italy
}

recovery and reuse of water, aiming at near-zero discharge of water by using closed-loop systems.

Up to now, much effort has been put in cleaning methods for waste water treatment (distillation, pervaporation, reverse osmosis, etc.). In contrast, there are always huge quantities of water emitted in the air by chemical plants, cooling towers, power plants, etc. The new stringent rules on emissions in the atmosphere limit the amount of vapor that can be discharged owing to the environmental concerns that these emissions imply in terms of greenhouse effects. Plume elimination system [5] is a consolidated technology developed since 1962 to limit the visibility and rainfall problems that usually occur close to cooling towers. This technology is based on a cooling process achieved through a close contact between water and air and a transfer of heat to the air by evaporation of a small amount of water. The heat is then dissipated through the hot air coming out of it. As a consequence, the dew point of the plume is reduced, dampening the problems related to water condensation and rain formation at the outlet of chimneys. Today, the

(c) The Author(s). 2019 Open Access This article is distributed under the terms of the Creative Commons Attribution 4.0 International License (http://creativecommons.org/licenses/by/4.0/), which permits unrestricted use, distribution, and reproduction in any medium, provided you give appropriate credit to the original author(s) and the source, provide a link to the Creative Commons license, and indicate if changes were made. The Creative Commons Public Domain Dedication waiver (http://creativecommons.org/publicdomain/zero/1.0/) applies to the data made available in this article, unless otherwise stated. 
separation and recovery of the water contained in the waste gaseous streams, on one hand is a constrain to be addressed, on the other can represent a new source of water. If industry can close the own water cycle by capturing evaporated water and therefore minimizing its request at a make-up level, for instance, more water can be made available also for other purposes. Jointly with water consumption, energy demand and its consumption are growing annually forcing power plants to produce more electrical and heat energy, which are connected with larger volumes of pollution gases. This causes the level of air pollution to be higher, especially in urban areas. Power plants as well as fertilizer factories, cement and steel factories, chemical and petrochemical industries are the main sources of air pollution, forming harmful chemical compounds such as $\mathrm{SO}_{\mathrm{x}}, \mathrm{NO}_{\mathrm{x}}, \mathrm{NH}_{3}$, $\mathrm{VOC}_{\mathrm{s}}$ unburned hydrocarbons, and particulate matter. These compounds are detrimental to human respiratory system and the environment, causing acid rain, stratospheric ozone depletion, photochemical smog, and a greenhouse effect.

Waste deposition in landfills can be considered as another important source of air pollution. In principle, the gaseous fraction generated by anaerobic digestion, socalled biogas, can be considered also a versatile renewable source, which can be used for replacement of fossil fuels in power production and in transport. However, it contains contaminants and pollutants $\left(\mathrm{H}_{2} \mathrm{~S}\right.$, siloxanes, halides, VFA) and necessarily needs pre-treatments before its use.

In this work, we analyze in detail the last advances achieved and the perspectives in the separation of water vapors from waste gaseous streams by using the "membrane condenser". This is a new membrane operation, recently introduced by Drioli and coworkers [6-10], which is finding more and more wide application in various fields related to vapor capture and which can be considered as a suitable alternative to conventional operations. Its aim is not limited on reducing the plume to avoid rainfall and visibility problems, but to recover the water vapour contained in waste gaseous streams. More recently, its use as pre-treatment stage for the reduction of contaminants contained in waste gaseous streams has proved increasingly promising, opening up new opportunities to use this technology. Here, we describe the operating principle, the main results achieved and the potential impact that its use could have in various industrial sectors.

\section{Membrane condenser: operating principle}

Membrane condenser is an innovative membrane operation, which employs microporous hydrophobic membranes as selective barriers for promoting water condensation (Fig. 1). The water contained in a saturated gas is condensed and recovered in the retentate side of the membrane module, thanks to the hydrophobic nature of the membrane and to the lower temperature at which the condenser is operated. The dehydrated gases, instead, pass through the membrane in the permeate side. When the water condenses in the membrane module the hydrophobic nature of the membrane prevents the penetration of the liquid into the pores.

The hydrophobic nature of the membranes not only avoids water droplets dragging, but also promotes vapors condensation exploiting the principle of dropwise condensation where, when condensation takes place on a surface that is not wet by the condensate, water beads up into droplets and rolls off the surface. Water vapor preferentially condenses on solid surfaces rather than directly from the vapor because of the reduced activation energy of heterogeneous nucleation in comparison to homogeneous nucleation. While the excess energy of

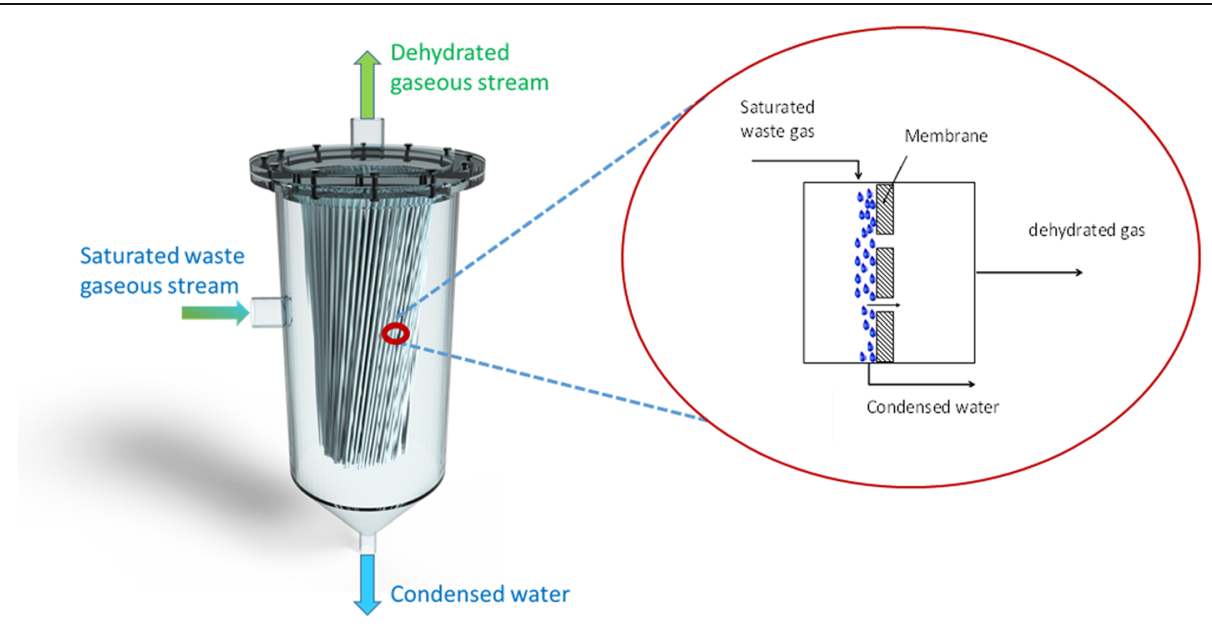

Fig. 1 Scheme of the membrane condenser process for the recovery of evaporated "waste" water from a gaseous stream 
a surface controls the heterogeneous nucleation process, it also determines the wetting behavior of the condensate, which has a significant impact on the overall performance [11]. The typical pore dimension of the membranes used is of $0.1-0.2 \mu \mathrm{m}$, well above the kinetic diameter of any gas present in the feed, therefore the membrane cannot have any selective separating function among the various gases as well as it will not be able to retain the water vapor that has not been condensed. Moreover, as described in details in [12], the proper design of the membrane module (e.g., membranes sealed only on the top side) allows a certain mobility to the hollow fiber membranes (that are shaken by the feed stream) and avoids the formation of liquid films over the membrane. Furthermore, the experimental measurements on the various tested membranes [12], - up today carried out at lab scale- lasted various months (also with stand-by periods), without showing remarkable performance variation, thus assuring their performance stability over the time.

In the condenser, the modulation of contact time between saturated stream and membranes as well as the control of temperature difference with the gaseous stream permit to control the fraction of contaminants that can be retained in condensed water and thus the concentration of contaminants that can be emitted with the dehydrated gas stream. This leads to a certain versatility of the system and means a further advantage with respect to other operation, since the retained contaminant can, in principle, (properly handled) be purified and reused.

\section{Current and potential applications}

Membrane condenser was initially developed in the framework of the FP7 project "CAPWA" [13], and it is now further technologically advanced and scaled up also in the EU H2020 project "MATChING" [14]. In both projects, membrane condenser was and is utilized only for water recovery from waste gaseous streams. A new application can be as pre-treatment stage to further separation units for minimizing the contaminants content of various gaseous streams, removing, in the meantime, part of the water vapor contained in gaseous streams. This aspect is sometime essential when the gas has to undergo further separation processes, like, for example, in carbon dioxide separation. Recent constraints and regulations on $\mathrm{CO}_{2}$ emissions from power plants have forced to focus on the separation of $\mathrm{CO}_{2}$ and to develop specific $\mathrm{CO}_{2}$ capture technologies that can be retrofitted to existing power plants as well designed into new plants with the goal to achieve $90 \%$ of $\mathrm{CO}_{2}$ capture limiting the increase in cost of electricity to no more than $35 \%$. Today membrane technology for separation and recovery of $\mathrm{CO}_{2}$ is a well-consolidated technique, mainly based on the use of polymeric membranes. Waste gaseous streams usually contain impurities such as water vapor, acid gases, olefins, aromatics and other organics. At relatively low concentrations, these impurities can cause membrane plasticization and loss of selectivity, while at higher concentrations they can condense on the membrane surface, which could be damaged.

Mass transport properties of polymeric membranes often used for waste gases treatment, such as $\mathrm{CO}_{2}$ separation, are significantly affected by the presence of water vapor, which usually competes with $\mathrm{CO}_{2}$ in permeation reducing its permeability as well as induces formation of water clusters, swelling etc. with consequent variation of permeabilities and selectivity [15-17]. The solution for a successful operation of polymeric modules is, also, a careful selection of feed pre-treatment. In this scenario, the possibility to use integrated membrane systems as alternative to traditional operations is becoming more and more attractive for gas purification. Analogously to what was done in the water purification field, where integrated membrane systems are today one of the leading technologies, also in the gas field, the necessity of pretreatment stages is a fundamental step both to fit required targets of contaminants content as well as to prolong the lifetime of the other downstream separation units. The reduction of water would significantly reduce the aforementioned phenomena, better exploiting the membrane area available and, thus, reducing the footprint of membrane GS units currently foreseen for the specific separation.

In this optic, membrane condenser can be a suitable pre-treatment stage placed before other separation technologies. By modulating the contact time between saturated stream and membrane condenser, it is possible to control the fraction of contaminants, which can be retained in condensed water. Although it is not always enough to reduce contaminants content below regulation limit (it mainly depends on $\Delta \mathrm{T}$ that can be imposed), however its use increases the operational time of the other pre-treatment stages such as activated carbons, scrubbers, etc. and can be used as pretreatment unit not only for membranes but also for other separation technologies such as PSA, cryogenic, absorption, etc. Moreover, the appropriate use of these systems would lead not only to reduce contaminants emissions and to recover water vapor contained in the gaseous stream, but also to recover high added value compounds such as VOC/VFA and/or to retain pollutants, which can be suspended in the waste gaseous stream.

Membrane condenser can be, thus, a key technology integrated in a system comprising other separation units for the treatment of the liquid and gaseous downstream. In the case of flue gas treatment, for example, the condensed water will contain contaminants, such as $\mathrm{SO}_{x}$ 
and eventually particulate, therefore, depending of their amount, it can be directly reused in the plant as make up or further treated by other purification technologies - not necessary based on membranes- obtaining high quality water (Fig. 2). The gaseous stream will contain $\mathrm{CO}_{2}, \mathrm{~N}_{2}, \mathrm{O}_{2}$ and traces of contaminants and water vapor which can be then further treated in a devoted device for separating and concentrating $\mathrm{CO}_{2}$. If the amount of contaminants or water vapor of this stream is not tolerable for the downstream unit used for $\mathrm{CO}_{2}$ separation, some pretreatments can be added in between. These latter will be significantly reduced in the size and their lifetime will be prolonged with respect to that foreseen without using condenser. The condensed water can contain salts (in particular when seawater is utilized as make-up water), biocides and algaecides (chlorine or $\mathrm{NH}_{3}$ ), in case of a cooling tower plume (Fig. 3). These latter are usually added to the cooling water to prevent growths of micro-organisms such as bacteria (e.g. legionella [18]), fungi and algae which are drifted together with the fine droplets emitted from the cooling towers. The emission in the atmosphere of these biocidal substances, such as chlorine and certain types of quaternary ammonium compounds, significantly affects the air quality in the areas near the plant; therefore, membrane condenser can be considered as an efficient pre-treatment unit able to both separate water vapor contained in waste gaseous streams, also retaining some of the most effective contaminants. The condenser can play an interesting role in the treatment of biogas (Fig. 4). Bio-methane is currently separated from $\mathrm{CO}_{2}$ using various technologies also including membranes. However, various are the pre-treatment stages that precede the membrane gas separation units, for preserving the membranes and meeting the specifications of grid injection. These pre-treatments are usually based on chillers, scrubbers for $\mathrm{H}_{2} \mathrm{~S}$ absorption with $\mathrm{Fe}_{2} \mathrm{O}_{3}$, activated carbons for siloxanes, mercaptans, etc.

Membrane condenser will allow separating water vapor and contaminants $\left(\mathrm{H}_{2} \mathrm{~S}, \mathrm{NH}_{3}\right.$, siloxanes and halides), VFA, VOCs, etc., from gaseous stream; condensed water can undergo a post-treatment to obtain high quality water, while VOC and VFA fraction can be recovered and further separated in other stages. As aforementioned, the use of the membrane condenser would imply a reduction of pre-treatment loads, which traduces in less solvent use, lower environmental impact, and improvement in air quality. Moreover, membrane condenser integrated with another separation units for VFA/ VOC recovery represent a novel highly selective and energy-efficient separation (pre-treatment) technology that will enhance the recovery of further resources from biodigester downstream, which can be then further purified and reused.

\section{Competing technologies}

As aforementioned, waste gases can contain various pollutants such as particulate matter, acid gases (like $\mathrm{SO}_{\mathrm{x}}, \mathrm{NO}_{\mathrm{x}}$, $\mathrm{HCl}$, etc.), green-house gases (such as $\mathrm{CO}_{x}, \mathrm{~N}_{x} \mathrm{O}_{y}$, parafluorocarbons, ozone depletion substances (such as Freon ${ }^{\mathrm{Tm}}$, Halon $^{\text {tw }}$ etc.), volatile organic compounds (such as toluene, xylene, trichloro-ethylene, trichloro- ethane, ethylene glycol mono ethyl ether [19]. ,Moreover, there can be large quantities of water vapor. The processes used for the treatment of waste gases, for eliminating the pollutants, are either physicochemical (such as scrubbing, chillers, adsorption, condensation, and oxidation) or biological.

Conventional physical-chemical treatments of polluted industrial waste gases, such as combustion or adsorption on activated coal filters, tend to waste a lot of energy

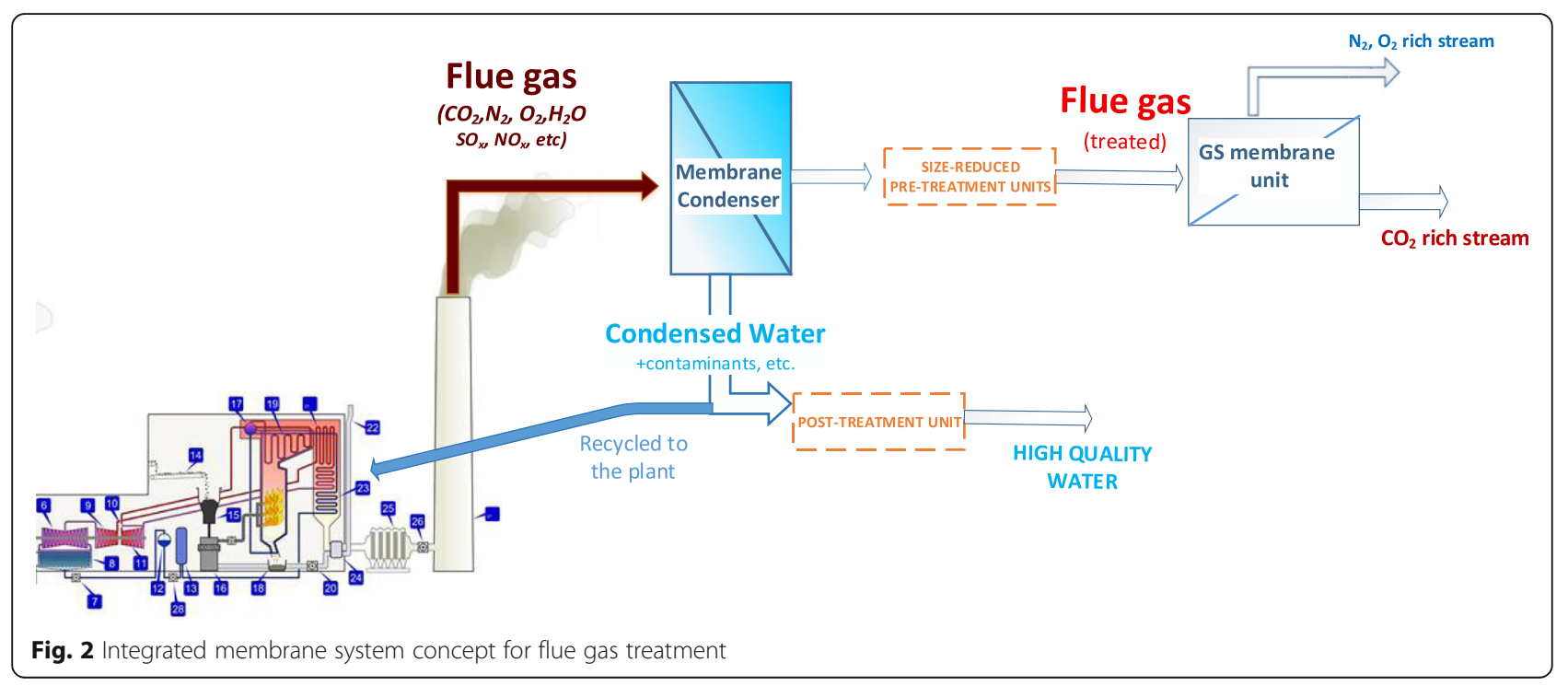






Fig. 3 Integrated membrane system concept for cooling tower plume treatment

and result in secondary pollution. Usually, bio-filters are used where organic compounds are degraded to carbon dioxide and water, while inorganic compounds, such as, Sulphur are oxidized to form oxygenated derivatives. Removal of the oxidized compounds from the media is an important consideration in the design of biofiltration systems. Often, for large air flows, biofilters are replaced by bio-scrubbers. In a bio-scrubber, the sprayed liquid is a suspension of microorganisms, which cycles back and forth between the spray chamber and a wastewater treatment unit where biodegradation takes place. They, however, can be used only for the removal of waste gases, which are sufficiently soluble because the mass transfer rate in a spray chamber is less than that attainable in a biofilter unit. In case the contaminant concentration in the outlet gas is too high, a second bio-scrubber inoculated with microorganisms capable of degrading lower contaminant concentrations must be installed.

Until now, the capture of evaporated water from gaseous streams has been carried out by condensation with heat exchangers [20], desiccants [21], dense membranes [22] or porous membranes [23].
The gas dehydration with dense membranes occurs via sorption-diffusion mechanism for vapor permeation [22, 24]. Even though the technical viability of this technology is confirmed by the results obtained, the high pressure necessary to promote the permeation of the water vapor through the membrane and the low pressure required on the permeate side specifically for hollow fiber membranes to avoid the condensation inside the fibers are the main disadvantage as it means vacuum pumps, high energy consumption and high costs. Desiccant drying system $[25,26]$ is another technique also explored by Siemens Power Generation, Inc. and the Energy \& Environmental Research Center at the University of North Dakota to remove water vapor from the power plant flue gas [27]. By designing and using a pilot-scale water extraction from turbine exhaust system, it was demonstrated that the system can remove $23-63 \%$ water vapor from flue gas by volume. However, a desiccant system has as disadvantages the regeneration of the desiccant and the low quality of the produced water.

Traditional condensers represent the easiest process for the capture of evaporated water from gaseous streams. However, corrosion phenomena due to the presence of

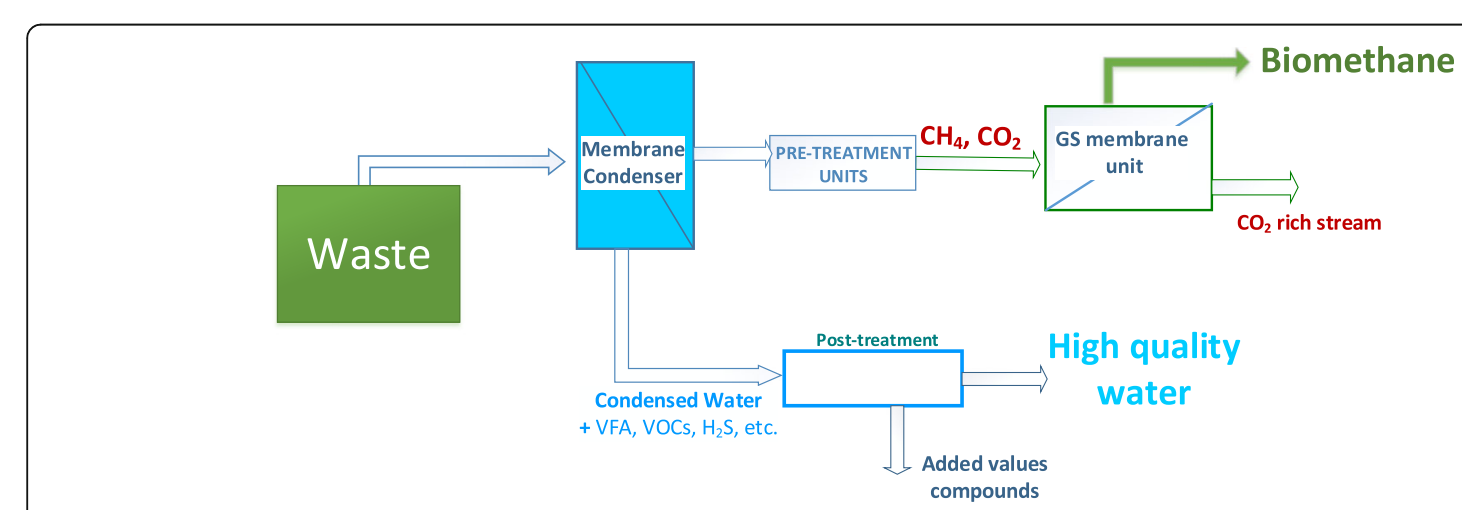

Fig. 4 Integrated membrane system concept for biogas treatment 
acid pollutant in the waste gases stream are their main limitation. Furthermore, with the same operating conditions, the heat-transfer area of a heat exchanger is more than $20 \%$ higher with respect to the membrane area necessary in a membrane condenser for obtaining the same amount of water. An example can be found by comparing the performance of membrane condenser with the results achieved by Jeong et al. [28], who developed an analytical model of heat and mass transfer processes in a flue gas condensing heat exchanger system. For the treatment of $172.52 \mathrm{~kg} / \mathrm{h}$ of a flue gas at $145^{\circ} \mathrm{C}$, Jeong et al. [28] calculated a heat-transfer area equal to $1.34 \mathrm{~m}^{2}$ for recovering the $42 \%$ of water. At the same conditions, a heat-transfer area of $1.03 \mathrm{~m}^{2}, 22.8 \%$ less is necessary in membrane condenser for obtaining the same water recovery.

More recently, transport membrane condenser were introduced [29-38]. Both hydrophilic and hydrophobic membranes can be used. In the transport membrane condenser with microporous hydrophilic membranes water vapor from gaseous stream condenses inside the membrane pores and passes through by direct contact with low-temperature water from the permeate side. In this way, the transported water is recovered along with virtually all of its latent heat. The conditioned flue gas leaves the transport membrane condenser at a reduced temperature and with a relative humidity below saturation. The clogging induced by the water condensed in the membrane pores inhibit the passage of non-condensable gases such as $\mathrm{CO}_{2}, \mathrm{O}_{2}, \mathrm{NO}_{\mathrm{x}}$, and $\mathrm{SO}_{2}$. The recovered water is of high quality and mineral free, and therefore can be used directly as boiler makeup water, as well as for other processes. Materials such as polyethersulphone, mixed cellulose ester (e.g., cellulose triacetate) and polyvinylchloride or ceramic are used in these types of hydrophilic membrane-based dehumidifiers.

Transport condenser with hydrophobic porous membranes are often used for simultaneous water and heat recovery from gas streams [39]. Water vapor contained in the gas stream passes through the membrane driven by its partial pressure difference and condenses into the cold water on the permeate side. This type of transport membrane condenser can be very effective in heat exchanging (i.e. heat recovery) but much attention should be paid to the undesirable condensation on the gas side or within the membrane pores.

Table 1 summarizes most relevant aspects of the three main different membrane technologies for water recovery.

\section{Membranes, module configurations and operating modes}

crucial aspects of membrane technology is to have available membranes with well-controlled properties, and the final performance of the process is a direct consequence of the structural and physicochemical parameters of the utilized membranes. This aspect, considerable for each membrane process, becomes fundamental for membrane condenser whose performance is intrinsically affected by the structure of the film in terms of thickness, porosity, mean pore size, pore distribution and geometry. The successful outcome of the process depends upon the capability of the membrane to combine high volumetric mass transfer (of the gaseous phase) with high resistance to liquid intrusion in the pores. Therefore, excellent mechanical properties, good thermal stability, excellent chemical resistance to feed solutions and high hydrophobicity are necessary to ensure the high liquid recovery factor at feed side. Since the hydrophobic character of the membrane is an essential requirement of a membrane condenser process, membranes have to be prepared from polymers with a low value of surface energy. Table 2 summarizes the main characteristics that membranes should have to be used in membrane condenser.

In general, two types of porous membranes can be used: (i) homogeneous hydrophobic and (ii) composite hydrophobic/hydrophilic membranes. In both cases, the membranes must be able to separate the contacting streams. Moreover, because hydrophobic membranes possess an inherent potential to be wetted by feed solute precipitation or adsorption onto the membrane surface, a reliable separation of the two phases have to be guaranteed at long-term operations. In particular, the characteristics needed for membranes are as follows:

1. High chemical stability. The chemical stability of the membrane material has a significant effect on its long-term stability. Any reaction between the feed and membrane material could possibly affect the membrane matrix and surface structure.

Streams with high load of acid gases are corrosive in the nature, which make the membrane material less resistance to chemical attack.

2. High thermal stability. Under high temperatures (up to $90^{\circ} \mathrm{C}$, typical in flue gas application), the membrane material may not be able to resist to degradation or decomposition. For operations at high temperatures, fluorinated polymers are good candidates owing to their high hydrophobicity and chemical stability.

3. High hydrophobicity in order to prevent wetting of the membrane pores.

4. Low fouling propensity because, in industrial applications, also gas streams can contain suspended particles that can cause plugging due to the small membrane pore size.

5. Long-term stability of the membrane which is important from an economic point of view for increasing the membrane life-time and for reducing membrane maintenance and operating costs. 


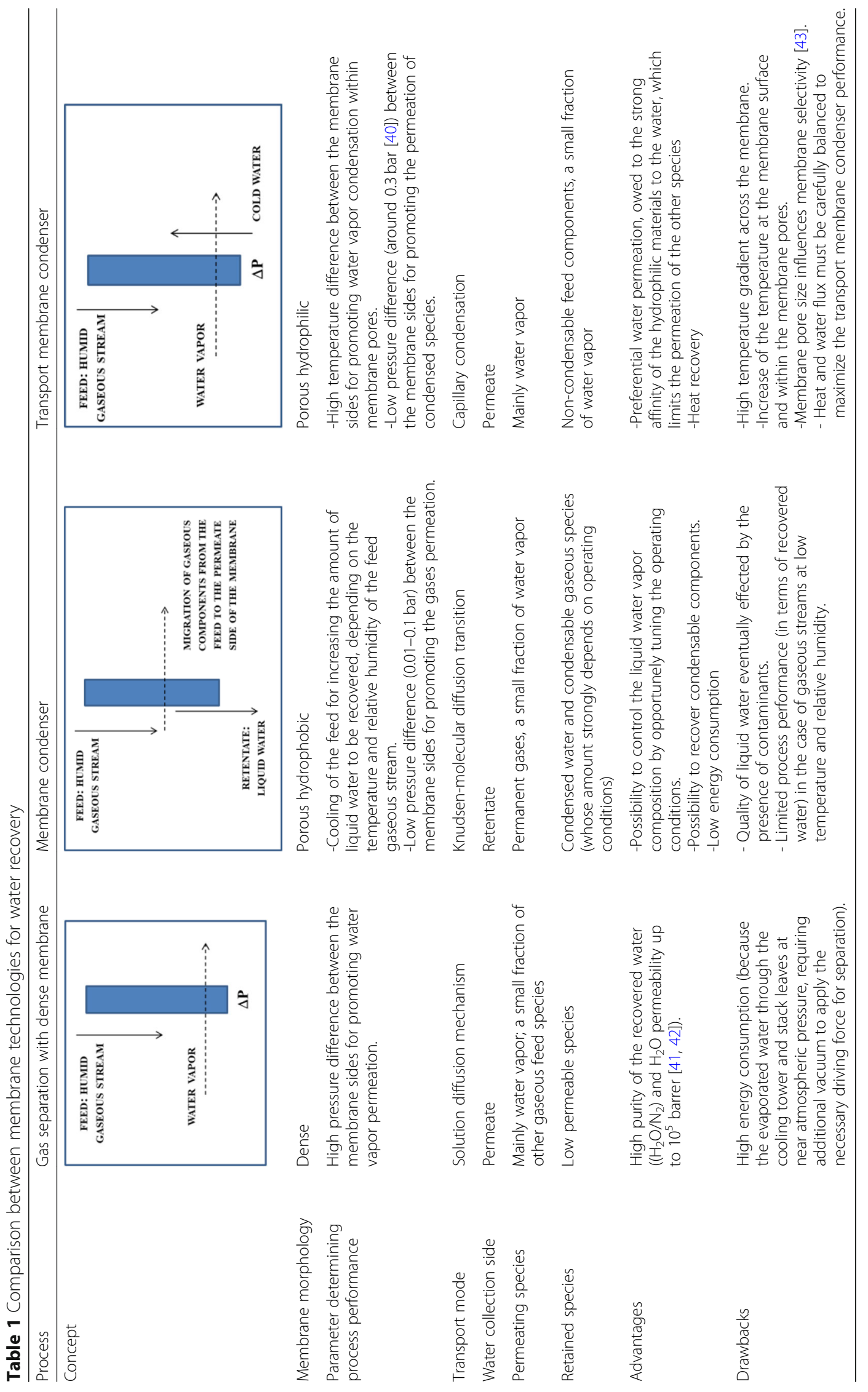


Table 2 Membrane characteristics

\begin{tabular}{|c|c|}
\hline \multirow{2}{*}{$\begin{array}{l}\text { Material } \\
\text { property }\end{array}$} & -hydrophobic \\
\hline & -resistant to alcohols, acids and surfactants \\
\hline Morphology & Porous, symmetric or asymmetric or composite \\
\hline Thickness & $20-100 \mu m$ \\
\hline Porosity & $70-80 \%$ \\
\hline $\begin{array}{l}\text { Thermal } \\
\text { conductivity }\end{array}$ & high \\
\hline Materials & $\begin{array}{l}\text { - Polymers (e.g., polypropylene, poly-vinylidenefluoride, } \\
\text { poly-tetrafluoroethylene, Hyflon AD (2,2,4-trifluoro-5- } \\
\text { tri-fluorometoxy-1,3-dioxole), ECTFE (Ethylene- } \\
\text { Chlorotrifluoroethlyene } \\
\text { copolymer), etc.)- } \\
\text { Modified hydrophobic ceramic membranes } \\
\text { (e.g., by coating hydrophobic polymethylsilsesquioxane } \\
\text { aerogels on alumina membrane supports, by applying } \\
\text { fluoroalkylsilanes hydrophobic agent on alumina hollow } \\
\text { fibers) } \\
\text {-mixed matrix (e.g., fillers of graphene, zeolite, carbon } \\
\text { nanotubes, etc.) }\end{array}$ \\
\hline Configuration & Flat, hollow fiber, tubular \\
\hline
\end{tabular}

\section{Low thickness and a high thermal conductivity in order to facilitate the heat transfer through the membrane.}

Commercial microporous hydrophobic membranes are available in capillary or flat-sheet forms and they were used in most of the membrane condenser experiments. In most of the them, membranes typically fabricated from polypropylene (PP) [12] or polyvinylidenedifluoride (PVDF) [6-8] were used, possessing a high porosity (70$80 \%)$, a membrane thickness of $10-300 \mu \mathrm{m}$ and pore sizes of about $0.2 \mu \mathrm{m}$.

Polypropylene has become one of the most used polymers for membrane synthesis, owing to its good thermal stability, chemical resistance, mechanical strength and low cost. PP is a linear polymer with structure consisting of $-\mathrm{CH}_{2} \mathrm{CH}\left(\mathrm{CH}_{3}\right)$ as the repeating unit. Depending upon the tacticity (arrangement of pendant groups along the backbone chain), it exists in semi-crystalline as well as amorphous forms. Isotactic PP, a semi-crystalline form, is the most used form for membrane synthesis at commercial scale. Isotactic PP has very good solvent resistance but still can be dissolved in strong solvents such as 1,2,4-trichlorobenzene, halogenated hydrocarbons, decalin, aliphatic ketones, xylene at relatively high temperatures. Three are the commonly used techniques for PP membrane fabrication including TIPS, stretching and tracketching. PVDF is a semicrystalline polymer with typical crystallinity between 35 to $70 \%$ and comprises of repeating units of $-\left(\mathrm{CH}_{2} \mathrm{CF}_{2}\right)_{n}{ }^{-}$. It has a glass transition temperature and melting point in the range of -40 to $-30{ }^{\circ} \mathrm{C}$ and 155 to $192^{\circ} \mathrm{C}$, respectively. Various homo and copolymers were used for PVDF membrane fabrication. It exhibits good mechanical strength, chemical resistance, thermal stability and reasonable aging resistance. Moreover, it is soluble in various common solvents such as $\mathrm{N}$-methyl-2-pyrrolidone, $\mathrm{N}, \mathrm{N}$-dimethyl acetamide and dimethyl formamide. Combination of good properties and easy processing makes PVDF the most widely applied material for membrane fabrication. PVDF is suitable for membrane preparation in various configurations including hollow fiber, flat sheet, nanofibers and tubular. In addition to these characteristics, the strong inherent hydrophobic character of PVDF provides the motivation for its use for membrane condenser membranes. PVDF membranes are usually synthesized through non-solvent induced phase separation process. In NIPS, PVDF is dissolved into a solvent at the room temperature, and then phase separation is induced through a non-solvent. Increasing interest arose in applying other techniques for synthesizing PVDF membranes, such as thermally-induced phase separation and electrospinning. TIPS is gaining interest due to several unique advantages including process simplicity, low tendency to defects formation, high porosity, reproducibility and ability to form narrow pore size distribution. In this process, PVDF is melt blended into an appropriate diluent and phase separation takes place by decreasing the temperature [44]. Electrospinning process is also gaining significant interest recently. It is a feasible process to produce sub-micron and nano-scale fibers. Compared to the NIPS techniques, electrospun membranes show superior void volume fraction (porosity), high specific surface area and high strength-to weight ratio [44]. Tailoring of characteristics (layer thickness, fiber diameter, porosity and functionality) of electrospun membranes can be achieved by changing electrospinning parameters, the material used and post processing treatment [45].

Hyflon, Ethylene-Chlorotrifluoroethylene copolymer (ECTFE) [10] and other amorphous perfluoro-polymers are excellent alternatives to the previous membranes due to their unique properties. Amorphous glassy perfluoropolymers such as Teflon $\mathrm{AF}^{\circ}$, Hyflon $\mathrm{AD}^{\circ}$ or Cytop ${ }^{\circ}$ are known for their good film forming properties and their high thermal and chemical stability. The latter is due to the very stable carbon-fluorine bond $(485 \mathrm{~kJ} / \mathrm{mol})$ in comparison with the carbon-carbon bond $(360 \mathrm{~kJ} / \mathrm{mol})$ [46]. The bulky substituted dioxole monomers in the structure of Teflon AF and Hyflon AD reduce the chain mobility and obstruct potential crystal formation, and in combination with the low cohesive energy of the fully fluorinated molecules this results in completely amorphous polymers with a poor packing and a high fractional free volume. The perfluorinated structure also gives these polymers a low tendency towards swelling, insolubility in common organic solvents and a strong hydrophobic character.

All such properties make them interesting for application as membranes in 'wet' gas treatment, where conventional polymers might suffer from plasticization by condensable species or from chemical attack in corrosive environment. 
An important role, in the operation of the membrane condenser is covered by the operational mode used. They can be:

1. Via an external coolant medium before entering the membrane module (Fig. 5a)

2. By a cold sweeping gas entering in the membrane module on the permeate side in counter-current with respect to the feed in order to decrease the temperature of the feed gas (Fig. 5b)

3. By a combination of the two modes mentioned above: the feed gas can be partially cooled via an external medium and then a sweeping gas is used for the final cooling of the stream (Fig. 5c).

The choice of the most appropriate operating mode mainly depends on the waste gaseous conditions (temperature, RH, etc.), on the final output to be reached and on the eventual downstream unit. The use of a sweep gas does not appear suitable when the condenser is used as a pre-treatment unit of a gas stream that requires further separations, such in the case of flue gas or biogas. The addition of a sweep gas, in fact, changes the composition of the gas stream, often diluting it, thus its use, for example in streams where $\mathrm{CO}_{2}$ has to be separated from the other gases by membrane gas separation devices would further reduce $\mathrm{CO}_{2}$ molar fraction and thus its driving force necessary for permeation, consequently lowering $\mathrm{CO}_{2}$ recovery and $\mathrm{CH}_{4}$ purity in the case of the biogas. Differently, it can be a suitable solution for treating the cooling tower plume, where no further separation is foreseen for the dehydrated gaseous fraction. In a recent work [9], we investigated and compared the performance of the different operating modes in terms of water recovery and energy consumption.

The configuration with external coolant resulted highly energy consuming with respect to the others with a water recovery of about $26 \%$, whereas that with cold sweep gas was the one with the lowest energy consumption (Fig. 6) and a very high recovery, obtained using a sweep flow rate
10 times greater than the feed one. The higher sweep flow rate in fact promoted the water recovery with a low energy consumption. The combination of the two cooling methods resulted a good compromise in terms of the water recovery of about $47 \%$ and energy consumption, which ranged between that of the other two configurations.

\section{Water recovery and quality}

The main variables driving the performance of the membrane condenser are:

- temperature of the plume

- relative humidity of the plume

- temperature difference between the plume and the condenser $(\Delta \mathrm{T})$

- feed flow rate/membrane area

As a general trend, the amount of water recovered as liquid in the retentate increases as much higher are the temperature and the relative humidity of the plume, the temperature difference between the plume and the condenser and as much lower is the feed flow rate/membrane area $\left(\mathrm{Q}^{\text {Feed }} / \mathrm{A}^{\text {Membrane }}\right)$. This latter ratio can be intended as an indication of the "contact time" of the feed flow rate with the active membrane area: a low value of this ratio means that the membrane area is more than enough to treat the feed; on the contrary, a high value of the ratio implies that the feed flow rate is too high with respect to the membrane area available in the module; as a consequence, the recovery will be lower.

For a set temperature of the plume, the amount of water that can be recovered increases as $\mathrm{RH}$ of the plume increases too and as the temperature of the condenser decreases (Fig. 7). In addition, as expected, the higher is the plume temperature, the higher is the fraction of water vapor that can be recovered, setting the other operating conditions. It is worth of note that, when the plume temperature is $90^{\circ} \mathrm{C}$, typical for a hot flue gas, a condenser operating at around $80^{\circ} \mathrm{C}$ is capable of recovering
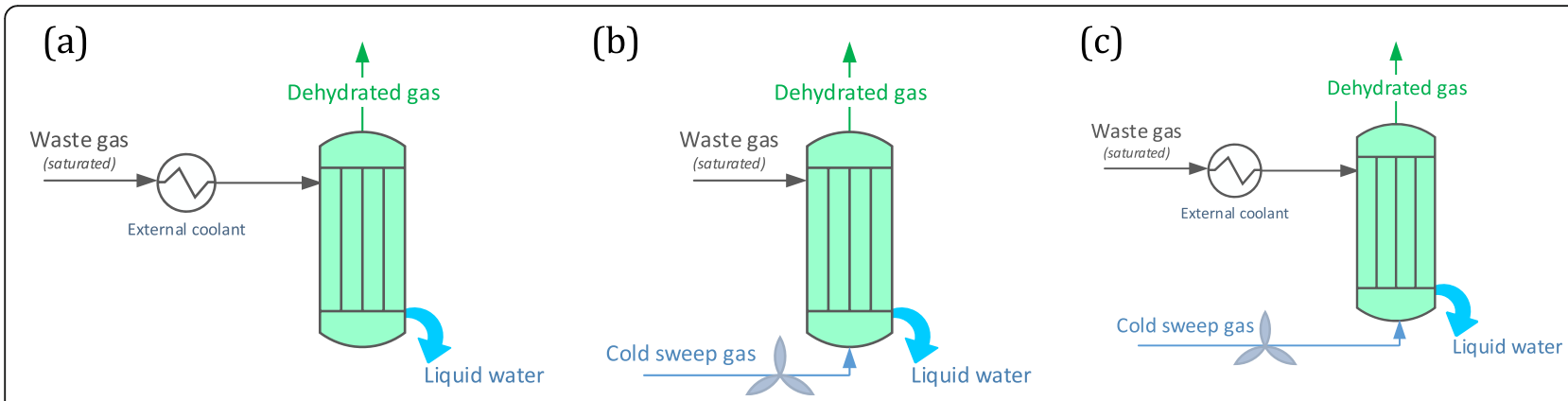

Fig. 5 Configurations of membrane condenser. Adapted with permission from Macedonio, F.; Brunetti, A.; Barbieri, G.; Drioli, E. Membrane condenser configurations for water recovery from waste gases. Sep. Pur. Tech., 181, 60-68, Copyright $\{2017\}$ of Elsevier 


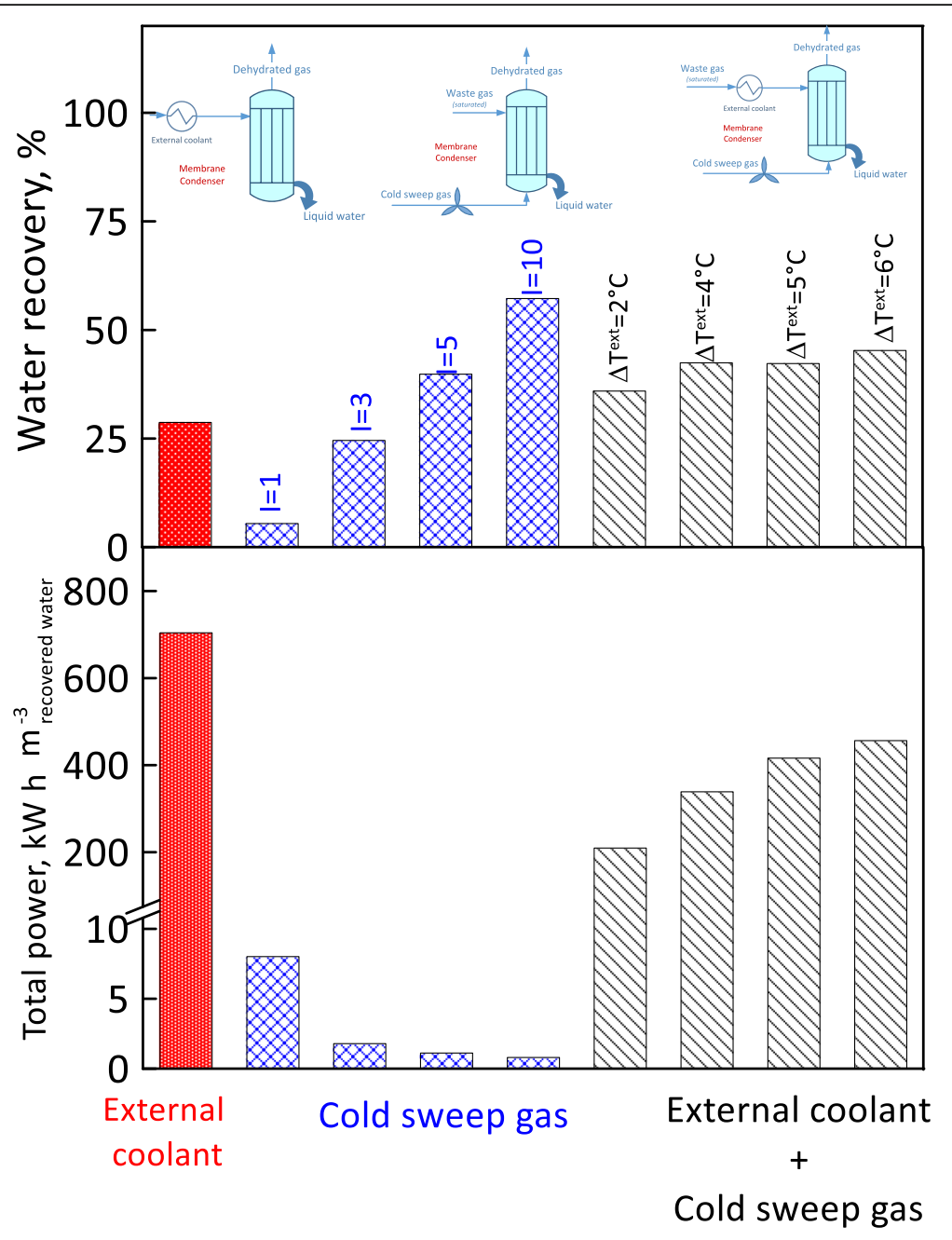

Fig. 6 Water recovery and energy consumption for the three configurations of membrane condenser. $\left(Q^{\text {Feed }}=30 \mathrm{Lh}^{-1}, \mathrm{RH}=100 \%, 55^{\circ} \mathrm{C}\right.$; sweep gas at $20^{\circ} \mathrm{C}$ ). Adapted with permission from Macedonio, F.; Brunetti, A.; Barbieri, G.; Drioli, E. Membrane condenser configurations for water recovery from waste gases. Sep. Pur. Tech., 181, 60-68, Copyright \{2017\} Elsevier

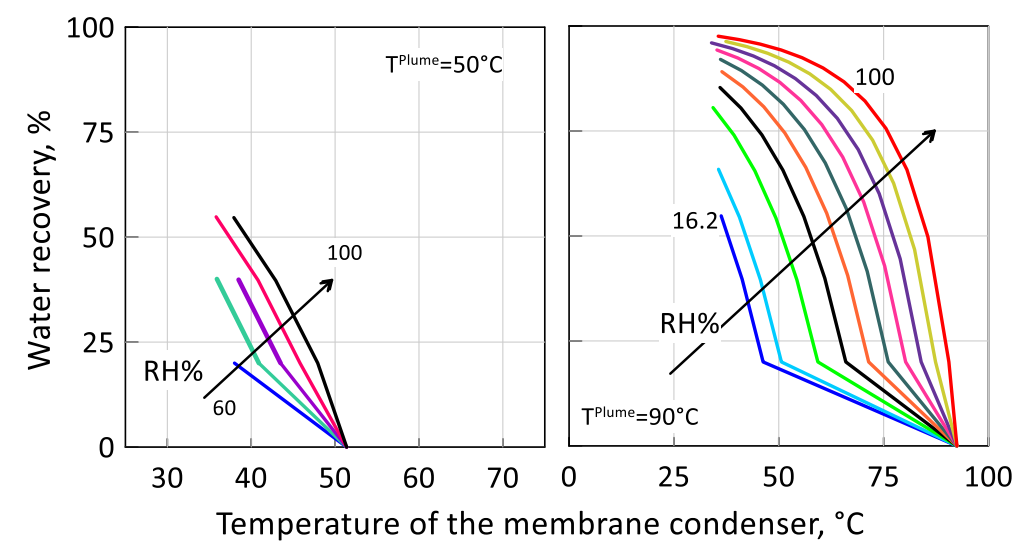

Fig. 7 Water recovery as a function of the temperature of the membrane condenser, at various $\mathrm{RH}$ of the feed. Adapted with permission from Macedonio, F.; Brunetti, A.; Barbieri, G.; Drioli, E. Membrane Condenser as a new technology for water recovery from humidified "waste" gaseous streams. Ind. Eng. Chem. Res., 52(3), 1160-1167, Copyright \{2012\} American Chemical Society 
about $20 \%$ of water, which is the amount necessary to make a plant self-supporting [47]. When the plume temperature is much lower, as in the case of cooling towers, the water that can be recovered in the condenser is much less but, anyhow, increases as much the condenser is cooled and as higher is the relative humidity of the feed (Fig. 8).

The energy consumption associated to a technology plays a key role in proposing it as a valid alternative to that well consolidated. The energy required for operating a membrane condenser is mainly ascribable to the heat duty required to condense the vapor or part of it. The energy consumption increases, as much higher is the amount of water that is recovered or, for a set water recovery as much higher are the initial temperature of the plume or the relative humidity, since this latter implies a greater amount of water in the feed (Fig. 8).

Up to now, the membrane condenser was experimentally investigated by using various types of hydrophobic membranes, such as PP [12], PVDF [6, 8], ECTFE [10], and feeding streams simulating the typical conditions either of flue gas or plume of a cooling tower. Figure 9 and Fig. 10 summarize the main results obtained as a function of the main operating conditions.

Figure 9 shows the water recovery by using commercial PVDF hollow fiber membranes (MEMBRANA, Germany) as function of $\mathrm{Q}^{\text {Feed }} / \mathrm{A}^{\text {Membrane }}$ and of the temperature difference between the plume and the condenser $(\Delta \mathrm{T})$. As expected, the water recovery increased with the $\Delta \mathrm{T}$. At a feed temperature of $55^{\circ} \mathrm{C}$, the condenser recovered more than $60 \%$ of water contained in the feed as vapor when it was cooled of about $15^{\circ} \mathrm{C}$. This recovery increased when the flue gas was at $65^{\circ} \mathrm{C}$ obtaining more than $65 \%$ as maximum value. The $\mathrm{Q}^{\text {Feed }}$ / $\mathrm{A}^{\text {Membrane }}$ exerted a key role in the performance of the condenser, especially at low $\Delta \mathrm{T}$. A high $\Delta \mathrm{T}$, instead, favors the water condensation compensating the low effect of a low residence time.

Innovative microporous hydrophobic membranes based on ECTFE were opportunely developed for membrane condenser application. These membranes, in flat sheet configuration, showed a very high porosity and hydrophobicity with respect to commercial PVDF membranes. Analogously to that, their performance was evaluated as a function of the main operating variables (Fig. 10). In all the cases, the membrane exhibited comparable performance to PVDF with a percentage of water recovered ranged between 35 to $55 \%$. The amount of water recovered with ECTFE membranes was similar to that of PVDF shown in Fig. 9, despite the lower mean velocity present in the ECTFE flat membrane module with respect to the PVDF membrane module. This is another confirmation of the higher productivity of ECTFE membranes.

Commercial PP hollow fibers were used in a module having $260 \mathrm{~cm}^{2}$ of membrane area for treating a gaseous stream simulating the plume of a cooling tower [12]. Plume temperature is relatively low with respect to that of flue gas, therefore 40 and $45^{\circ} \mathrm{C}$ were considered for the experiments. At a set value of $\mathrm{Q}^{\text {Feed }} / \mathrm{A}^{\text {Membrane }}$, the water recovery increased with increasing the $\Delta \mathrm{T}$ between the module and the plume (Fig. 11). It passed from around $10 \%$ measured with a $\Delta \mathrm{T}$ of $3{ }^{\circ} \mathrm{C}$ to more than $40 \%$ with $11{ }^{\circ} \mathrm{C}$ of temperature difference.

Figure 12 summarizes the water recovery obtained with the different membranes and in the various operating conditions aforementioned. As it can be seen, the temperature difference between the waste gaseous stream and the membrane condenser is the principal variable to drive membrane performance. Even though some variations can be obtained in dependence on the membrane, feed temperature and $\mathrm{Q}^{\mathrm{Feed}} / \mathrm{A}^{\text {Membrane }}$, the water recovery follows a linear trend, which increases with $\Delta \mathrm{T}$.
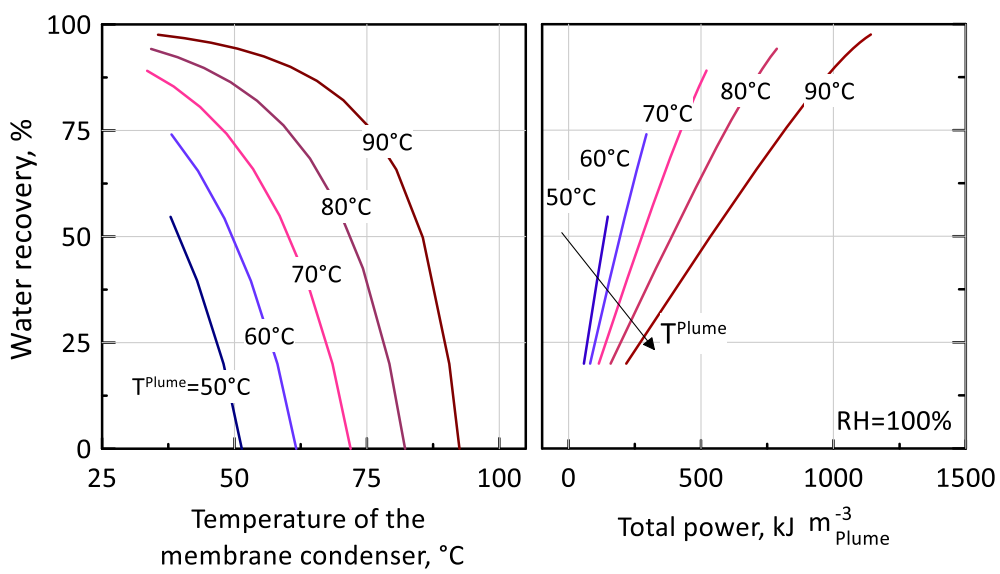

Fig. 8 Water recovery as a function of the temperature of the membrane condenser and of the total power required at various plume temperatures. Adapted with permission from Macedonio, F.; Brunetti, A.; Barbieri, G.; Drioli, E. Membrane Condenser as a new technology for water recovery from humidified "waste" gaseous streams. Ind. Eng. Chem. Res., 52(3), 1160-1167, Copyright \{2012\} American Chemical Society 

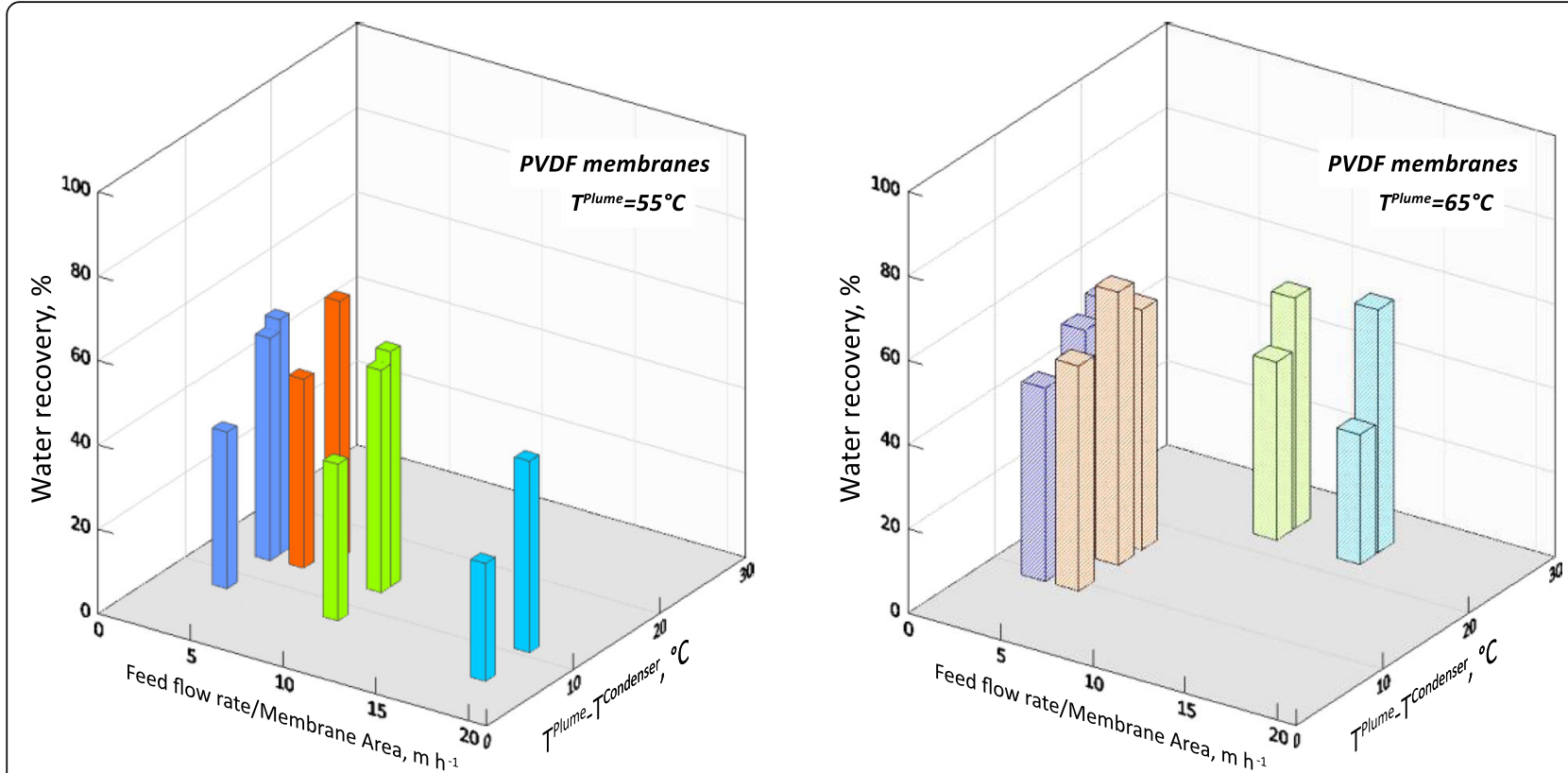


and the condenser

\section{Contaminants}

As reported in Section 2, membrane condenser can be used as pre-treatment unit for removing part of contaminants contained in the waste gaseous stream before treating it in other separation units. In our previous works $[7,12]$, we investigated the capability of membrane condensers to control, by opportunely tuning the operating conditions, the condensation of contaminants



Fig. 10 Water recovery obtained with ECTFE membranes as a function of the $Q^{\text {Feed }} / A^{\text {Membrane }}$ and the temperature of the plume (e.g., $\mathrm{SO}_{\mathrm{x}}, \mathrm{NH}_{3}, \mathrm{HF}$ ) in the recovered liquid water. The amount of contaminants that can be retained in the condensed water is directly proportional to the initial concentration of the contaminants in the feed and increases with the temperature difference between plume and condenser (Fig. 13). The retaining of these species in the liquid water is based on the Henry's law, therefore their solubility in aqueous solution increases reducing the

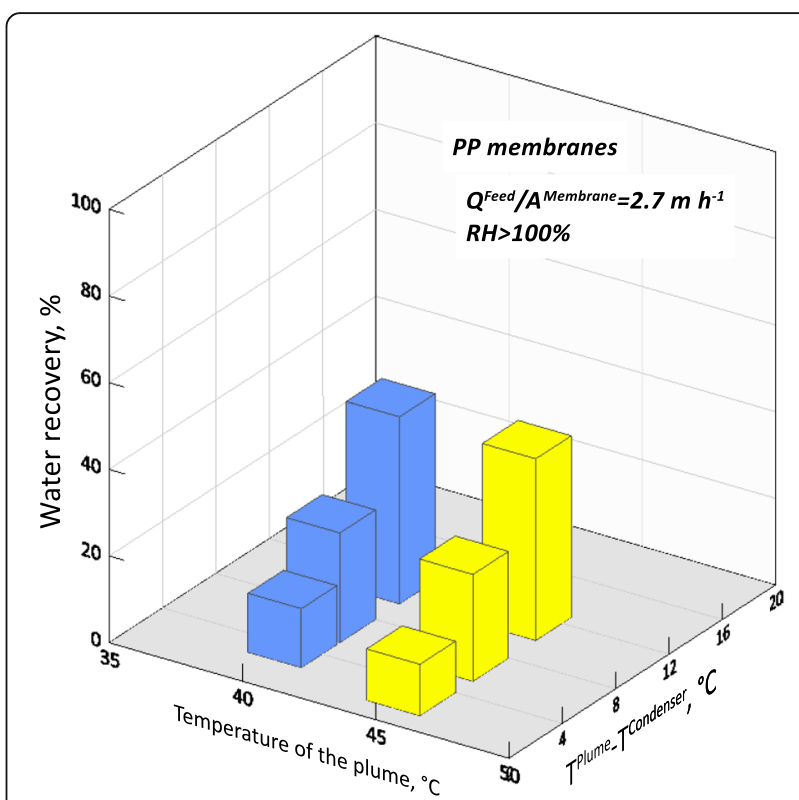

Fig. 11 Water recovery obtained with PP membranes as a function of the temperature of the plume and the temperature difference between the plume and the condenser 




Fig. 12 Water recovery as a function of the temperature difference between the plume and the condenser

temperature of the membrane module. Clearly, for a fixed concentration of contaminant in the feed, the amount of $\mathrm{SO}_{\mathrm{x}}, \mathrm{NH}_{3}$, or HF that is removed from gaseous stream depends also on the nature of the contaminant and, thus, on its Henry's constant. The concentration of $\mathrm{SO}_{2}, \mathrm{HF}$ and $\mathrm{NH}_{3}$ in the recovered

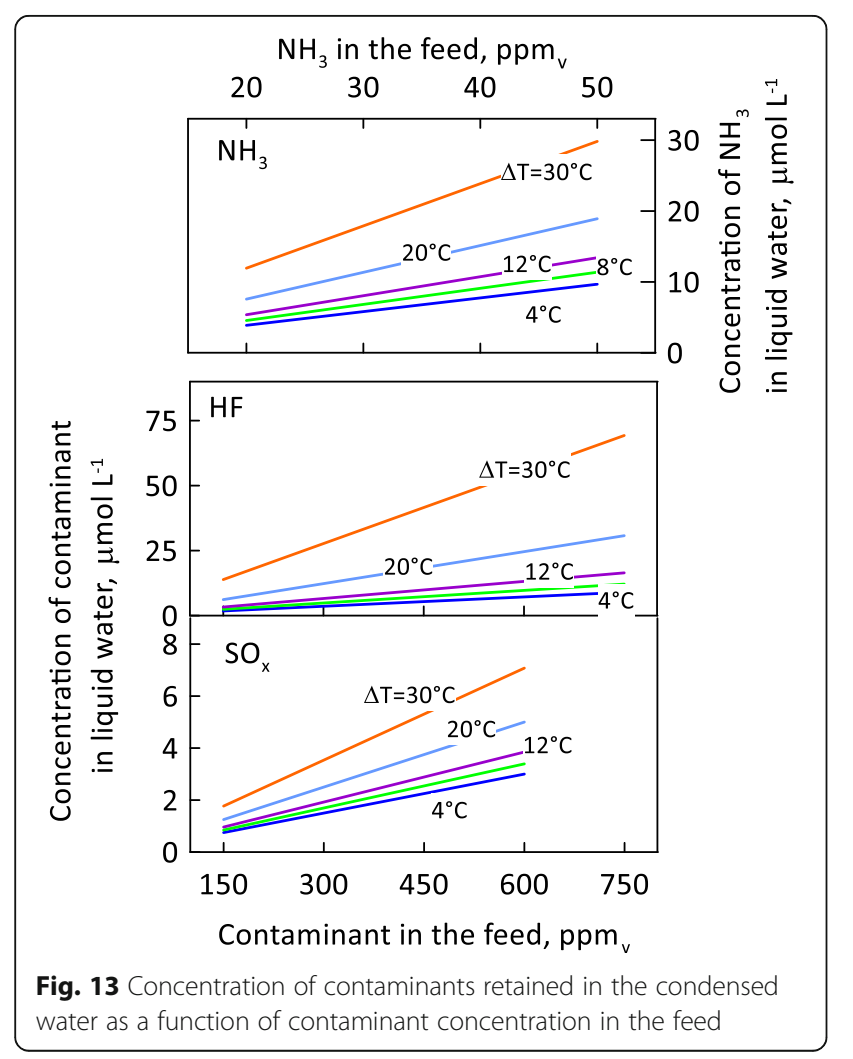

liquid water was found to be completely different, with a higher condensation of $\mathrm{NH}_{3}$, as it is characterized by a higher solubility in water. With a $\Delta \mathrm{T}$ of $30^{\circ} \mathrm{C}$, around $60 \%$ of $\mathrm{NH}_{3}$ contained in the plume was retained in the liquid water. The condenser was less efficient in recovering $\mathrm{SO}_{\mathrm{x}}$ and $\mathrm{HF}$, owing their lower solubility water. Anyhow, their concentration in the recovered water was well below the World Health Organization recommended limits [48] of $1.6 \mathrm{ppm}$ for $\mathrm{HF}$ and $0.2 \mathrm{ppm}$ for $\mathrm{SO}_{2}$.

The use of the membrane condenser as effective unit for pre-treating gaseous stream partially retaining contaminants was also confirmed with devoted experiments carried out in different operating conditions by using PVDF [7] and PP membranes [12] (Fig. 14). Independently of the membrane type, the amount of $\mathrm{NH}_{3}$ retained in the condensed water increased as much higher was the temperature difference between the gaseous stream and the membrane condenser. In the case of PP membranes, the higher initial concentration of $\mathrm{NH}_{3}$ in the feed led to a greater amount of $\mathrm{NH}_{3}$ recovery, which reached up to $57 \%$ with a $\Delta \mathrm{T}$ of $11.5^{\circ} \mathrm{C}$. Another important parameter affecting the concentration of contaminants in the liquid water was the $\mathrm{Q}^{\mathrm{Feed}} / \mathrm{A}^{\mathrm{Mem}-}$ brane, whose increase led to a decrease of the amount of $\mathrm{NH}_{3}$ retained in condensed water. This trend can be attributed to the fact that as much higher is the $\mathrm{Q}^{\mathrm{Feed} /} \mathrm{A}$ Membrane, lower is the "contact time" between the membrane and the gaseous stream, thus less water is retained as liquid and, as a consequence, also less contaminants.

\section{Conclusions}

Water shortage is now pushing towards the exploitation of unconventional sources, among which water vapor contained in waste gaseous streams plays a major role. In the last few years, membrane condenser was introduced as a "tailor-made" innovative membrane operation



Fig. 14 Concentration of $\mathrm{NH}_{3}$ retained in condensed water as a function of temperature difference between the plume and the membrane condenser for PVDF and PP membranes 
for the exploitation of this source, where microporous hydrophobic membranes are used as selective barriers also able to promote water condensation. In dependence on the gaseous stream considered (biogas, flue gas, etc.) and of its final utilization, this water can be directly reused in the plant or further purified and used for other purposes.

The capability of retaining water from waste gaseous streams such as flue gas, biogas, plume of cooling tower, etc., subsequently opened to other perspectives of use of this technology, proposing membrane condenser also as a highly efficient pre-treatment stage of (waste) gaseous streams. By modulating the contact time between saturated stream and membranes in condenser unit and opportunely regulating the temperature difference between condenser and waste gaseous stream, it is possible to modulate the fraction of contaminants, which can be retained in condensed water. It is important to notice that the membrane condenser, even not always enough to reduce contaminants content below regulation limit, allows to increase the operational time of other pretreatment stage such as adsorbent beds (desiccants, etc.) or absorption liquids.

When the gaseous streams require further separation for recovering a defined specie, such as the flue gas for $\mathrm{CO}_{2}$ capture, biogas for biomethane production, etc., if these separation stages are membrane-based, the reduction of water content allows a better performance of the separation unit, depleting phenomena such as formation of water clusters, swelling etc. that usually affect polymeric membranes used, for example in $\mathrm{CO}_{2}$ separation, with consequent reduction of permeabilities and selectivity. Analogously, the membrane condenser would result a suitable pre-treatment placed before cryogenic distillation or PSA. In some cases, the controlled water condensation offered by the membrane condenser will allow also the recovery of valuable compounds such as VFA, VOCs, salts, which usually can be dragged in the gaseous phase.

Although the use of membrane condenser has grown rapidly, the fulfillment of these ambitious objectives is strictly connected to the development of suitable hydrophobic porous membranes specifically targeting at membrane condenser process. The membranes used up to now were originally used for microfiltration and ultrafiltration. They are available for analyzing the membrane condenser process, but they are not the ideal membrane materials. It is important and interesting to develop specific membranes with desired properties and structures targeted for membrane condenser application. High hydrophobicity, excellent chemical resistance and stability toward the industrial waste gases, narrow distribution of pore size and improved structural and morphological characteristics are the fundamental aspects to be further investigated. Moreover, all feasible approaches devoted to enhance mass transfer phenomena, control fouling problems, and related drawbacks (clogging, loss of hydrophobicity, etc.) are expected to be matter of further investigations for researchers working in this area.

\section{Abbreviations \\ A: Area; ECTFE: Ethylene-Chlorotrifluoroethlyene; GS: Gas Separation; P: Pressure, Pa; PP: Polypropylene; PSA: Pressure Swing Adsorption; PVDF: Polyvinylidene fluoride; $\mathrm{Q}$ : Volumetric feed flow rate, $\mathrm{m}^{3} \mathrm{~h}^{-1}$; $\mathrm{RH}$ : Relative Humidity; T: Temperature, ${ }^{\circ} \mathrm{C}$; VFA: Volatile Fatty Acids; VOC: Volatile Organic Compounds}

\section{Acknowledgements}

Not applicable.

\section{Authors' contributions}

$A B$ and FM performed data analysis. $A B$ and FM wrote the manuscript with support from GB. ED conceived the original idea. AB supervised the project. All authors read and approved the final manuscript.

Funding

Not applicable.

\section{Availability of data and materials}

Data sharing not applicable to this article as no new datasets were generated or analysed during the current study.

\section{Competing interests}

The authors declare no conflict of interest.

Received: 24 May 2019 Accepted: 29 July 2019

Published online: 12 September 2019

References

1. http://www.fao.org/nr/water/aquastat/water_use/index.stm (Last access: 19/ 03/2019).

2. Resource efficiency and low carbon economy, Annual Indicator Report Series (AIRS) European Environment Agency, 2016. https://www.eea.europa. eu/airs/2016/resource-efficiency-and-low-carboneconomy (Last access: 19/ 03/2019).

3. https://www.spire2030.eu/sites/default/files/pressoffice/spire-roadmap.pdf (Last access: 19/03/2019).

4. http://ec.europa.eu/environment/action-programme/index.htm(Last access: 19/03/2019).

5. http://www.seas.columbia.edu/earth/wtert/sofos/nawtec/1968-NationalIncinerator-Conference/1968-National-Incinerator-Conference-26.pdf (Last accessed: 09 July 2019).

6. Macedonio F, Brunetti A, Barbieri G, Drioli E. Membrane condenser as a new technology for water recovery from humidified "waste" gaseous streams. Ind Eng Chem Res. 2012;52(3):1160-7.

7. Macedonio F, Cersosimo M, Brunetti A, Barbieri G, Drioli E. Water recovery from humidified waste gas streams: quality control using membrane condenser technology. Chem Eng Proc Proc Int. 2014;86:196-203.

8. Brunetti A, Santoro S, Macedonio F, Figoli A, Drioli E, Barbieri G. Waste gaseous streams: from environmental issue to source of water by using membrane condensers. Clean - Soil, Air, Water. 2014:42(8):1145-53.

9. Macedonio F, Brunetti A, Barbieri G, Drioli E. Membrane condenser configurations for water recovery from waste gases. Sep Pur Tech. 2017;181: 60-8.

10. Drioli E, Santoro S, Simone S, Barbieri G, Brunetti A, Macedonio F. Figoli, A. ECTFE membrane preparation for recovery of humidified gas streams using membrane condenser. React Funct Polym. 2014;79:1-7.

11. Enright R, Miljkovic N, Al-Obeidi A, Thompson CV, Wang EN. Condensation on Superhydrophobic surfaces: the role of local energy barriers and structure length scale. Langmuir. 2012;28(40):14424-32.

12. Macedonio F, Frappa M, Brunetti A, Barbieri G, Drioli E. Water and contaminants recovery from plume of cooling tower. Env. Eng. Res., 2019 (Accepted). 
13. Capture of evaporated water with novel membranes, GA n² 246074, http://www.watercapture.eu/ (Last access: 08 July, 2019).

14. Materials \& Technologies for Performance Improvement of Cooling Systems performance in Power Plants, GA n 686031, http://matching-project.eu/ (Last access: 08 July, 2019).

15. Scholes CA, Freeman BD, Kenthish SE. Water vapour permeability and competitive sorption in thermally rearranged (TR) membranes. J. Mem. Sci. 2014;470:132-7.

16. Brunetti A, Cersosimo M, Dong G, Woo KT, Lee J, Kim JS, Lee YM, Drioli E, Barbieri G. In situ restoring of aged thermally rearranged gas separation membranes. J. Mem. Sci. 2016;520:671-8.

17. Cersosimo M, Brunetti A, Drioli E, Dong G, Woo KT, Lee J, Lee YM, Barbieri G. Separation of $\mathrm{CO} 2$ from humidified ternary gas mixtures using thermally rearranged polymeric membranes. J Mem Sci. 2015;492:257-62.

18. Cooling Tower Institute. Legionellosis. Guideline: Best Practices for Control of Legionella. Cooling Tower Institute; July 2008. Available from: https:// www.cti.org/downloads/WTP-148.pdf . (Last access: 19/03/2019).

19. Kuniko U, Chang J-S. Removal of volatile organic compounds from air streams and industrial flue gases by non-thermal plasma technology. IEEE Trans Dielectr Electr Insul. 2000:602-14.

20. Michels B, Adamczyk F, Koch J. Retrofit of a flue gas heat recovery system at the Mehrum power plant. An example of power plant lifetime evaluation in practice. In: Proceedings of POWER-GEN Europe conference; 2004. p. 10-1.

21. Folkedahl B, Weber GF, Collings ME. Water extraction from coal-fired power plant flue gas, final report, DOE cooperative agreement no. DE-FC2603NT41907. Pittsburgh, PA: National Energy Technology Laboratory; 2006.

22. Sijbesma H, Nymeijer $K$, van Marwijk R, Heijboer R, Potreck J, Wessling M. Flue gas dehydration using polymer membranes. J. Mem. Sci. 2008; 313:263-76.

23. Wang T, Yue M, Qi H, Feron PHM, Zhao S. Transport membrane condenser for water and heat recovery from gaseous streams: performance evaluation. J Membr Sci. 2015:484:10-7.

24. Shamu A, Miedema H, Borneman Z, Nijmeijer K. Dehydration of supercritical carbon dioxide using dense polymeric membranes: a techno-economical evaluation. Sep Purif Technol. 2019;224:209-18.

25. Liu XH, Zhang Y, Qu KY, Jiang Y. Experimental study on mass transfer performances of cross flow dehumidifier using liquid desiccant. Energy Convers Manag. 2006:47(15-16):2682-92.

26. Zurigat YH, Abu-Arabi MK, Abdul-Wahab SA. Air dehumidification by Triethylene glycol desiccant in a packed column. Energy Convers Manag. 2004;45(1):141-55

27. Copen JH, Sulliva TB, Folkedahl BC, Carney B. Principles of flue gas water recovery system. In: Proceedings of the power-gen international conference. Las Vegas NV; 2005.

28. Jeong K, Kessen MJ, Bilirgen H, Levy EK. Analytical modeling of water condensation in condensing heat exchanger. Int J Heat Mass Transf. 2010; 53:2361-8.

29. Yan S, Zhao S, Wardhaugh L, Feron PHM. Innovative use of membrane contactor as condenser for heat recovery in carbon capture. Environ Sci Technol. 2015;49:2532-40.

30. Gao D, Li Z, Zhang H, Chen H, Wang L, Liu H. The investigation of desulphurization and water recovery from flue gas using ceramic composite membrane. Int J Energy Res. 2019;43:1747-59.

31. Zhang X, Jia L, Peng Q, Dang C. Experimental study of condensation heat transfer in a condenser with a liquid-vapor separator. Appl Therm Eng. 2019; 152:196-203.

32. Xie J, Xu J, Liang C, She Q, Li M. A comprehensive understanding of enhanced condensation heat transfer using phase separation concept. Energy. 2019;172:661-74.

33. Yang $\mathrm{B}, \mathrm{Chen} \mathrm{H}$. Heat and water recovery from flue gas: application of micro-porous ceramic membrane tube bundles in gas-fired power plant. Chemical Engineering \& Processing: Process Intensification. 2019;137:116-27.

34. Tu T, Cui Q, Liang F, Xu L, He Q, Yan S. Water recovery from stripping gas overhead $\mathrm{CO} 2$ desorber through air cooling enhanced by transport membrane condensation. Sep Purif Technol. 2019;215:625-33.

35. Yan S, Cui Q, Tu T, Xu L, He Q, Feron PHM, Zhao S. Membrane heat exchanger for novel heat recovery in carbon capture. J Membr Sci. 2019; 577:60-8

36. Yang B, Shen $G$, Chen H, Feng Y, Wang L. Experimental study of condensation heat-transfer and water-recovery process in a micro-porous ceramic membrane tube bundle. Appl Therm Eng. 2019;155:354-64.
37. Dan G, Zhaohao L, Heng Z, Haiping C, Chao C, Kai L. Moisture and latent heat recovery from flue gas by nonporous organic membranes. J Clean Prod. 2019;225:1065-78.

38. Zhao S, Yan S, Wang DK, Wei Y, Qi H, Wu T, Feron PHM. Simultaneous heat and water recovery from flue gas by membrane condensation: experimental investigation. Appl Therm Eng. 2017;113:843-50.

39. Zhao S, Feron PHM, Xie Z, Zhang J, Hoang M. Condensation studies in membrane evaporation and sweeping gas membrane distillation. JMembr Sci. 2014;462:9-16.

40. Kim JF, Park A, Kim SJ, Lee P, Cho Y, Park H, et al. Harnessing clean water from power plant emissions using membrane condenser technology. ACS Sustain Chem Eng. 2018;6(5):6425-33

41. Metz S, Van de Ven W, Potreck J, Mulder M, Wessling M. Transport of water vapor and inert gas mixtures through highly selective and highly permeable polymer membranes. J Membr Sci. 2005:251(1):29-41.

42. Vane L, Namboodiri V, Lin G, Abar M, Alvarez F. Preparation of waterselective polybutadiene membranes and their use in drying alcohols by pervaporation and vapor permeation technologies. ACS Sustain Chem Eng. 2016:4(8):4442-50

43. Wang D, Bao A, Kunc W, Liss W. Coal power plant flue gas waste heat and water recovery. Appl Energy. 2012;91(1):341-8.

44. Liu F, Hashim NA, Liu Y, Abed MRM, Li K. Progress in the production and modification of PVDF membranes. J. Memb. Sci. 2011;375:1-27.

45. Tijing LD, Choi J, Lee S, Kim S, Kyong H. Recent progress of membrane distillation using electrospun nano fi brous membrane. J Memb Sci. 2014; 453:435-62.

46. Jansen JC, Friess K, Drioli E. Organic vapour transport in glassy perfluoropolymer membranes: a simple semi-quantitative approach to analyze clustering phenomena by time lag measurements. J. Memb. Sci. 2011;367:141-51.

47. Feeley TJ, Pletcher S, Carney B. McNemar. Power-Gen International: A.T. Department of Energy/National Energy Technology Laboratory's Power Plant Water R\&D Program; 2006.

48. https://www.who.int/news-room/fact-sheets/detail/ambient-(outdoor)-airquality-and-health (Last access: 19/03/ 2019).

\section{Publisher's Note}

Springer Nature remains neutral with regard to jurisdictional claims in published maps and institutional affiliations.
Ready to submit your research? Choose BMC and benefit from:

- fast, convenient online submission

- thorough peer review by experienced researchers in your field

- rapid publication on acceptance

- support for research data, including large and complex data types

- gold Open Access which fosters wider collaboration and increased citations

- maximum visibility for your research: over $100 \mathrm{M}$ website views per year

At $\mathrm{BMC}$, research is always in progress.

Learn more biomedcentral.com/submissions 